\title{
Quality Characteristics and Moisture Mobility of Giant Salamander (Andrias davidianus) Jerky during Roasting Process
}

\author{
Wengang Jin $\left(\mathbb{D},{ }^{1}\right.$ Xiaohua Chen, ${ }^{1}$ Jingzhang Geng, ${ }^{1}$ Jing Jin, ${ }^{1}$ Jinjin Pei, ${ }^{1}$ \\ Ruichang Gao $\mathbb{D}^{1,2}$ and Dejing Chen $\mathbb{D}^{1}$ \\ ${ }^{1}$ Bio-Resources Key Laboratory of Shaanxi Province, School of Biological Science and Engineering, \\ Shaanxi University of Technology, Hanzhong 723001, China \\ ${ }^{2}$ College of Food and Biological Technology, Jiangsu University, Zhenjiang 212013, China
}

Correspondence should be addressed to Wengang Jin; jinwengang@nwafu.edu.cn and Ruichang Gao; xiyuan2008@ujs.edu.cn Received 10 March 2021; Accepted 23 June 2021; Published 1 July 2021

Academic Editor: Luis Patarata

Copyright (C) 2021 Wengang Jin et al. This is an open access article distributed under the Creative Commons Attribution License, which permits unrestricted use, distribution, and reproduction in any medium, provided the original work is properly cited.

Quality attributes and water mobility of giant salamander jerkies (GSJs) during roasting process $\left(160^{\circ} \mathrm{C}\right.$, for $0,20,40,60$, and 80 min) were investigated. The results showed that $a^{*}, b^{*}$ values and shear force increased of GSJs roasting from 20 to 80 min, while $L^{*}$, yield, and moisture content decreased significantly $(p<0.05)$. Sensory assessment showed that GSJs at a roasting time of $40-60 \mathrm{~min}$ had higher scores. GSJs contained great amount of healthy unsaturated fatty acids (including DHA and EPA), and the total amino acids and essential amino acids were among $59.33-71.77 \mathrm{~g} \cdot 100 \mathrm{~g}^{-1}$ and $25.94-31.40 \mathrm{~g} \cdot 100 \mathrm{~g}^{-1}$, respectively. The mobility of the immobilized moisture and free moisture were shrunk dramatically during roasting. The proton density weighted images also exhibited the moisture shrinkage during roasting. In addition, $T_{22}$ and $T_{23}$ were positively correlated with MRI signal, moisture content, and yield of GSJs, but negatively correlated with shear force and overall acceptability, respectively. Thus, in view of various quality attributes and sensory evaluation, a roasting time of 40-60 min was favored for nutritive GSJs production. LF-NMR and MRI might be employed to profile the quality characteristics during roasting as a rapid and nondestructive analytical tool.

\section{Introduction}

Giant salamander (Andrias davidianus) lives as the biggest amphibian on planet Earth, mainly populated in central and south China $[1,2]$. Dwelling on planet Earth for approximately 350 million oldness, giant salamander had once been overexploited, due to commercial interests for foods, nutraceutical, and medicines, and it was by that hold as an extinct wildlife [2,3], Currently, man-made reproducing and farming of Andrias davidianus have been industrialized in some provinces of China, such as Shaanxi, Hunan, Henan, and Guizhou [3,4]. In view of its increasing quantity and decreasing price, much effort upon utilization approaches of cultured Andrias davidianus has been made to increase its added value [4]. To date, bioactive peptides [5-7], cutting meat $[8,9]$, collagen or gelatin $[4,10-12]$, chondroitin sulfate [13], edible oil [14], and cooked dishes [15] from giant salamander have been previously reported.
Jerky is an intermediate-moisture convenience food fascinated by consumers worldwide [16]. Generally, sliced muscles of beef, pork, chicken, and fish have been roasted to manufacture jerky products. Silva et al. found that chicken jerky suffered acute oxidation and quality deterioration after storage at retail conditions [17]. Han et al. pointed out that curing methods would impact the quality characteristics of jerky [18]. Several reports also found that processing conditions (cooking methods, roasting temperature, and time) would affect processing yield, color, shear force, water activity, and volatile compounds of jerky [19-22]. Therefore, the physicochemical and quality characteristics of jerky are very critical for the final product quality control.

Roasting, a common practice during jerky processing, involves both mass and heat transfer. Roasting can endow jerky with favorable traits and the moisture dehydration of raw meat, depending on heat intensity and processing time [22]. Moisture migration and distribution are inevitable 
during jerky roasting, and their characterizations might be helpful for product quality control $[19,23]$. These years, lowfield nuclear magnetic resonance (LF-NMR) has been broadly used as a nondestructive technique for depiction of moisture distribution and migration in agricultural products processing, because of its agility, swiftness, nondestructiveness, and cheapness $[24,25]$. Principle of LF-NMR chiefly laid on the determination of resonant radio frequency assimilation when imposed to a foreign fixed magnetic device. Spin-spin transverse relaxometry detection $\left(T_{2}\right)$, coupled with $T_{2}$ opposite contour spectrum, is deemed to produce signal of moisture level, environmental attributes of moisture, and communication of moisture with the nearby macromolecules [24]. Magnetic resonance imaging (MRI), the second form of NMR relaxometry, can offer visualized signal of water migration and distribution $[25,26]$. Up to now, LF-NMR and MRI techniques enjoy wide application in food quality characterization, especially quantification the variations of moisture allotment and movability involved in food processing and storage [27, 28].

In the field of processing of jerky-like products, Li et al. [29] studied the variations in moisture movability of chicken jerky after dehydrating with LF-NMR technique and found that acute interactions were spotted between moisture content, shear force, and LF-NMR signals. Song et al. [25] reported that the moisture changes in dehydrated abalone could be characterized by LF-NMR signals, and a simulation equation of water level was made employing the acute correlation between LF-NMR signals and water levels. Cheng et al. [24] pointed that LF-NMR and MRI facilities might be adopted to notice the water movability and allotment of Pacific oyster after dehydrating. These studies showed that LF-NMR and MRI facilities were potential tools for fast assay of the moisture dynamics and distribution of semidried products, and they might be used for quality control when combined with physicochemical and nutritional attributes [26, 27].

In the context of exploitation and utilization of farmed giant salamander, the use of its meat for jerky production could increase its added value and commercial product variety, yet little literature about the preparation and quality characteristics of giant salamander jerky could be found. The aim of present study was to explore the influence of roasting process upon the quality characteristics and water mobility of giant salamander jerkies (GSJs) during roasting. Besides, the nutritional and microstructure of the resulting jerky were also reported.

\section{Materials and Methods}

2.1. Raw Materials and Ingredients. Five frozen giant salamanders (approximately $2.57 \pm 0.41 \mathrm{~kg}$ ) were purchased from Hanzhong Dragon Head Mountain Aquatic Co., Ltd. (Shaanxi, China) and kept in a commercial refrigerator (CF50 Dometic Group, Shenzhen, China) at $-20^{\circ} \mathrm{C}$ before use. Several condiments such as cooking wine, prickly ash, ginger, onion, sugar, salt, and pepper were bought from a local supermarket (Hanzhong, China), sealed with plastic bags, and stored at $4^{\circ} \mathrm{C}$ for 5 days in a chromatography cabinet (GSP, Yiran Refrigerator Co., Ltd., Beijing, China).

2.2. Preparation of Giant Salamander Jerky. Frozen giant salamander carcasses were first thawed with running water at room temperature $\left(22^{\circ} \mathrm{C}\right)$, washed clean, sliced into around 250 bar-like samples (approximately $3 \mathrm{~cm} \times 2 \mathrm{~cm} \times 1 \mathrm{~cm}$ ), and weighed. These samples were cured and deodored in a complex seasoning containing cooking wine, prickly ash, ginger, onion, sugar, salt, and pepper for 90 min. After draining juice, 250 sliced samples were roasted in a multifunction oven (Rational SCC61, Germany) for preparation of control (40 jerky samples) and roasted jerky (200 samples, 50 for each roasting time). The roasting temperature was set at $160^{\circ} \mathrm{C}$ for various time intervals $(20$, 40,60 , and $80 \mathrm{~min}$ ), and samples without roasting were used as control $(0 \mathrm{~min})$. Then, jerky samples of each roasting time $(0,20,40,60$, and $80 \mathrm{~min})$ were cooled to room temperature, put into polyethylene bags, and sealed in a vacuum packing machine (DZ-600/2S, Tianjin Food Machinery Co., Ltd., Zhucheng, China), respectively. All jerky samples were subjected to the following measurement.

2.3. Yield Determination. The jerky yield was calculated according to a modified method of Kim et al. [30]. Briefly, 5 jerky samples at each roasting time (20, 40, 60, and $80 \mathrm{~min})$ were weighed, respectively, before and after roasting, and yield was expressed by the percentage of weight before and after each roasting time, using the following formula:

$$
\text { yield }(\mathrm{g} / 100 \mathrm{~g})=\frac{\text { sample weight after roasting }(\mathrm{g})}{\text { sample weight before roasting }(\mathrm{g})} \times 100 \text {. }
$$

2.4. Measurement of Shear Force. The shear force of 10 prepared jerky specimens after each roasting time $(0,20,40$, 60 , and $80 \mathrm{~min}$ ) was measured through a TA-XT2i Texture Analyzer (Stable Micro Systems, Godalming, UK), according to an adjusted method of Yang et al. [31]. The jerky samples (cooled to room temperature) were dressed evenly before test. Apparatus conditions were adjusted as follows: Warner-Bratzler blade probe, pretest velocity: $2.0 \mathrm{~mm} / \mathrm{s}$, test velocity: $2.0 \mathrm{~mm} / \mathrm{s}$; posttest velocity: $8.0 \mathrm{~mm} / \mathrm{s}$; shear depth: $15 \mathrm{~mm}$; and induction load: $5 \mathrm{~g}$.

2.5. Chromatism Assay. The surface chromatism of jerky samples was evaluated by a premium colorimeter $(\mathrm{NH}$ Technical Co., Ltd., Shenzhen, China). The CIELAB color system was used to obtain the parameters of $L^{*}$ (lightness), $a^{*}$ (redness), and $b^{*}$ (yellowness), prior to standardization with a white calibration plate. Three samples for each roasting time were prepared for color analysis, and six surface positions of single specimen were selected to get color parameters as mean. 
2.6. Assay of Moisture Content. Jerky samples (5 for control and 5 for each roasting time) were assayed in a blast air oven (Hebi Metallurgical Machinery Equipment Co., Ltd., Henan, China) at $105^{\circ} \mathrm{C}$ until constant weight, according to GB5009.3-2016 (National Food Safety Standard of China). The moisture level was calculated by the mass variation before and after dehydrating [25].

2.7. Fatty Acid Determination. Fatty acid analysis of jerky samples (3 for control and 3 for each roasting time) was performed by gas chromatography (Agilent Technologies 7890A, USA) equipped with flame ionization detection and capillary DB-Wax column $(30 \mathrm{~m} \times 0.25 \mathrm{~mm}$ id $\times 0.25 \mu \mathrm{m}$ film thickness, Agilent Technologies, USA). Each fatty acid in the chromatogram was detected by contrasting the retention times with standard mixes (FAME Mix Supelco, Bellefonte, PA, USA). The content of each fatty acid was calculated using the peak area normalization method by gas chromatography software. The detailed procedure and instrumental parameters were reported previously [32].

2.8. Amino Acid Analysis. Hydrolyzed using a $6 \mathrm{~mol} \cdot \mathrm{L}^{-1} \mathrm{HCI}$ solution at $105^{\circ} \mathrm{C}$ for $24 \mathrm{~h}$, amino acid compositions of jerky specimens ( 3 for control and 3 for each roasting time) were performed by HPLC (Thermo Fisher Scientific Co., USA), installed with an amino acid kit reported previously [4]. Alkaline hydrolysis was also performed for the determination of tryptophan level.

2.9. Microstructure Analysis. The jerky specimens (3 for control and 3 for each roasting time) were dipped in $3 \%$ glutaraldehyde at $4^{\circ} \mathrm{C}$ for $12 \mathrm{~h}$ and then lyophilized in a freeze-drier (Sihuan Scientific Co., Ltd., Beijing, China). After this treatment, the microstructural photos of jerky samples were captured in a scanning electric microscopy (SEM) (Phenom Pure, Shanghai, China), at a magnification of $230 \mathrm{x}$ and $5 \mathrm{kV}$ voltage.

2.10. Sensory Assessment. Sensory assessment of the jerky specimens was performed by the quantitative descriptive analysis (QDA) and the Hedonic test, according to a modified approach described previously [33, 34]. Ten panelists with no rhinitis and smoking ( 5 males and $5 \mathrm{fe}$ males, age of 22-32) were recruited from Meat Laboratory of Department of Food Science, Shaanxi University of Technology. Three training sessions were held to familiarize the panelists with the aim, detailed experimental steps, and requirements of sensory assessment before serving jerky samples at various roasting times. Cold jerky samples (5 for control and 5 for each roasting time) were sliced before serving, respectively. The samples of different roasting times were finally served to panelists in a random order. Sensory assessment conditions included room temperature around $23-25^{\circ} \mathrm{C}$ and humidity of 50-54\%, and filament lamp (36 W) was employed. Each assessor provided their scores for appearance, flavor, and texture through a 9-point descriptive scale (1.0-3.0, weak; 4.0-6.0, medium; and 7.0-9.0, strong).
The value of 1.0 corresponded to the lowest score for each attribute and 9.0 to the highest. On the hedonic scale, 1.0-3.0 was deemed as not acceptable, 4.0-5.0 fairly acceptable, 6.07.0 good (acceptable), and 8.0-9.0 very good.

2.11. LF-NMR and MRI Trial. The $T_{2}$ transverse relaxation trials were carried out in a NMR analyzer (HT-MRSI20-40A; Huantong Instrumental Corporation, Shanghai, China). The resonance frequency and magnetic field intensity of the device was 23.2 MHz and 0.5 T. The jerkies (5 for control and 5 for each roasting time) were put into a hydrostatic glass column, and a $40 \mathrm{~mm}$ diameter radio frequency loop was employed to acquire Carr-Purcell-Meiboom-Gill sequence decline information, with $90^{\circ}$ pulse time of $24 \mu \mathrm{s}$ and $180^{\circ}$ pulse time of $48 \mu \mathrm{s}$, respectively. The repetition time between two scans was $1.2 \mathrm{~ms}$, and the other parameters included the number of echos $=2048$, number of iterations $=10$, number of points $=200$, and signal-to-noise ratio $=300$.

Magnetic resonance images (MRI) were also captured on the NMR instrument with the imaging software (Vs-Ultimate, Huantong Instrumental Corporation, Shanghai, China). Proton density weighted images were captured by the above NMR imaging procedure. The following scanning conditions were used: view field $=256 \mathrm{~mm} \times 256 \mathrm{~mm}$, resonant frequency $=23.2 \mathrm{MHz}$, pulse interval $=1$, and repetition time $=20$. The final images and the corresponding signal intensity after normalization was obtained by Image Pro software.

2.12. Statistical Approach. Obtained data were displayed as mean \pm standard deviation $(n \geq 3)$. Figures are plotted through Origin Pro 2018 (OriginLab Co., USA). The acute variation was performed through ANOVA Tukey's tests by IBM Statistics SPSS 24 (SPSS Inc., USA).

\section{Results and Discussion}

3.1. Quality Attributes as Influenced by Roasting Time. The present study monitored several quality parameters (processing yield, shear force, moisture content, and color values) of giant salamander jerkys (GSJs) during roasting process (Figure 1). The processing yield changes of GSJs prepared at different roasting times are shown in Figure 1(a). The yield of GSJs significantly decreased with roasting time $(p<0.05)$. In contrast with raw $(0 \mathrm{~min})$, processing yield of giant salamander meat roasted for $20 \mathrm{~min}$ decreased to $74.36 \%$. The yield continued diminishing with the increase of roasting time. A lower yield of $30.05 \%$ was found when roasted for $80 \mathrm{~min}$ in the present study. This yield trend was mainly caused by water evaporation during roasting, as cooking loss of meat products occurred commonly during food processing $[16,21]$. Low processing yield could enhance product quality and storage stability, but it sometimes harmed the economic interest of manufactures. Similar results of beef jerky fried at different temperatures were also reported by Liu et al. [23].

Figure 1(b) profiles the moisture proportion of GSJs with different roasting times. The initial moisture content of giant 


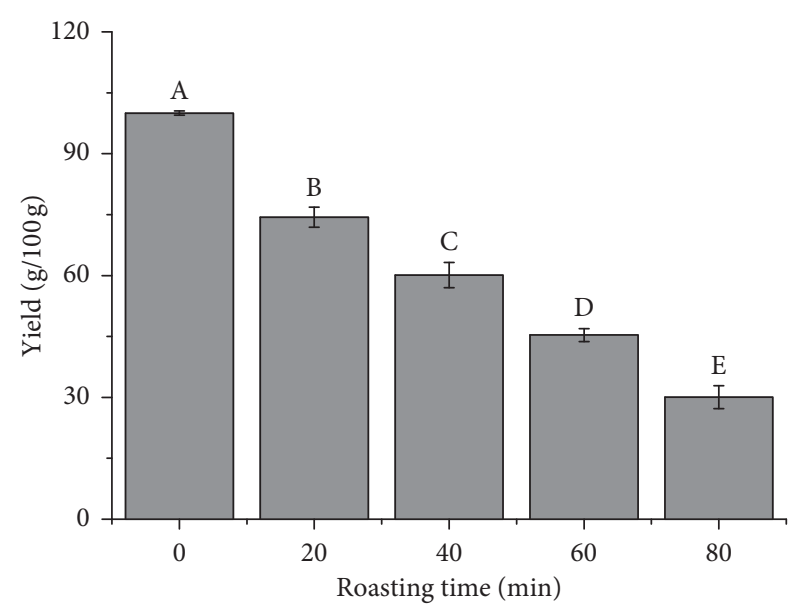

(a)

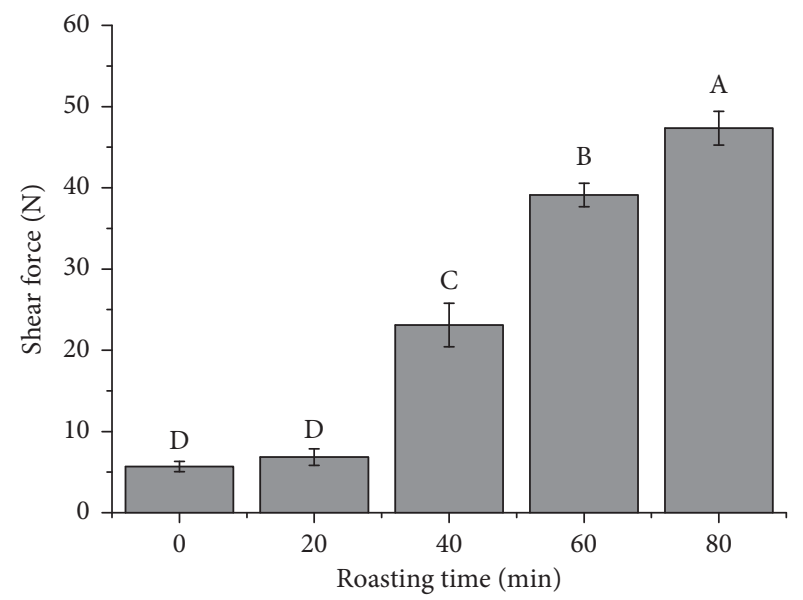

(c)

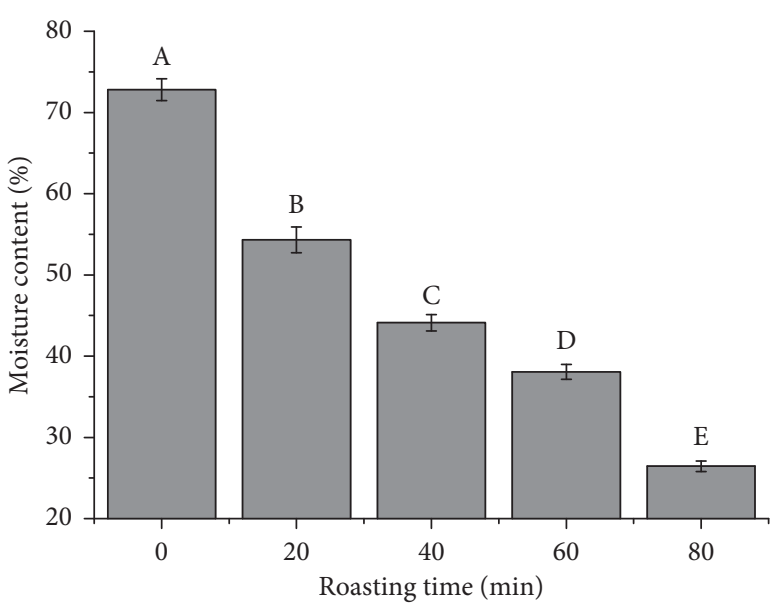

(b)

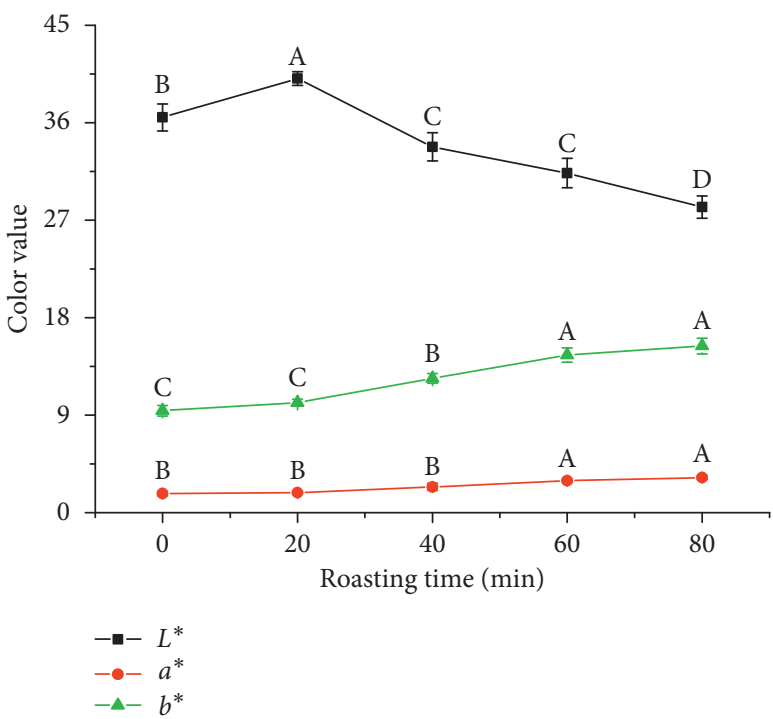

(d)

Figure 1: Quality attributes as influenced by roasting time. (a) Processing yield; (b) moisture content; (c) shear force; (d) color values. All data were reported as mean \pm standard deviation $(n \geq 3)$. Different lowercase letters indicate significant differences $(p<0.05)$.

salamander meat sample before roasting $(0 \mathrm{~min})$ was $72.81 \pm 1.35 \%$, lower than that of Veal $(75.26 \%)$ and Hashi (76.44\%), but higher than that of Noeimi $(64.12 \%)$ and Najdi $(71.82 \%)$ [35]. With the increase of roasting time (20-80 $\mathrm{min})$, the moisture content of GSJs decreased significantly from $54.33 \pm 1.58 \%$ to $26.44 \pm 0.65 \% \quad(p<0.05)$ (Figure 1(b)), owing to water evaporation caused by heat transfer during roasting $[36,37]$.

Shear force is a common quality indicator of meat products, which can reflect the texture of meat. Figure 1(c) shows the shear force of GSJs after various roasting times. No sharp variation for shear force of GSJs was found when roasted for 0 and $20 \mathrm{~min}(p>0.05)$, although a slight increase was observed at $20 \mathrm{~min}$. The shear force value increased sharply when roasted above $40 \mathrm{~min} \quad(p<0.05)$. Through roasting, the water proportion of giant salamander jerky decreased with increasing roasting time, and myofibrillar proteins suffered severe denaturation and crosslinking, leading to crust hardness of dried meat [36-38].

Surface color of meat jerky products is a critical attribute for consumers' food choice. The instrumental color parameters of GSJs with different roasting times are shown in Figure $1(\mathrm{~d})$. The $L^{*}$ value of GSJs after roasting $20 \mathrm{~min}$ were greater than initial raw jerky $(0 \mathrm{~min})(p<0.05)$, whereas $a^{*}$ and $b^{*}$ parameters slightly fluctuated $(p>0.05)$. Once roasting, raw meat protein denatured gradually because of heat stress, leading to the increase of $L^{*}$ value. With the extension of roasting time from 40 to $80 \mathrm{~min}$, the $L^{*}$ value (lightness) decreased significantly, while $a^{*}$ (redness) and $b^{*}$ (yellowness) values increased gradually $(p<0.05)$. This is because moisture escaped from jerky samples during roasting; some browning compounds may be produced through Maillard reaction between protein, amino acid, and carbonyl of giant salamander's muscle [39]. A similar color 
TABLE 1: Sensory score of giant salamander jerky as affected by roasting time.

\begin{tabular}{lcccc}
\hline Roasting time (min) & Color & Flavor & Texture & Overall acceptability \\
\hline 0 & $\mathrm{np}$ & $\mathrm{np}$ & $\mathrm{np}$ & $\mathrm{np}$ \\
20 & $5.35 \pm 0.87^{\mathrm{c}}$ & $4.35 \pm 0.46^{\mathrm{b}}$ & $4.02 \pm 0.21^{\mathrm{c}}$ & $4.02 \pm 0.15^{\mathrm{c}}$ \\
40 & $7.22 \pm 1.02^{\mathrm{a}}$ & $6.22 \pm 0.31^{\mathrm{a}}$ & $6.22 \pm 0.36^{\mathrm{b}}$ & $7.36 \pm 0.22^{\mathrm{a}}$ \\
60 & $7.86 \pm 0.55^{\mathrm{a}}$ & $7.36 \pm 0.72^{\mathrm{a}}$ & $6.87 \pm 0.20^{\mathrm{a}}$ & $7.94 \pm 0.31^{\mathrm{a}}$ \\
80 & $6.41 \pm 0.43^{\mathrm{b}}$ & $7.12 \pm 0.30^{\mathrm{a}}$ & $7.02 \pm 0.43^{\mathrm{a}}$ & $6.28 \pm 0.28^{\mathrm{b}}$ \\
\hline
\end{tabular}

Data were expressed as mean \pm standard deviation of three determinations. Different lowercase superscripts in the same column denote significant differences $(p<0.05)$. np: not performed.

TABLE 2: Fatty acid profiles of giant salamander jerkies as affected by roasting time.

\begin{tabular}{|c|c|c|c|c|c|}
\hline \multirow{2}{*}{ Fatty acids } & \multicolumn{5}{|c|}{ Content (mg.100 g $\left.\mathrm{g}^{-1}\right)$} \\
\hline & $0 \mathrm{~min}$ & $20 \mathrm{~min}$ & $40 \mathrm{~min}$ & $60 \mathrm{~min}$ & $80 \mathrm{~min}$ \\
\hline C12:0 & $0.70 \pm 0.00^{\mathrm{d}}$ & $2.23 \pm 0.01^{\mathrm{a}}$ & $1.11 \pm 0.01^{\mathrm{b}}$ & $0.91 \pm 0.01^{c}$ & $0.90 \pm 0.02^{c}$ \\
\hline $\mathrm{C} 13: 0$ & $0.70 \pm 0.00^{\mathrm{d}}$ & $1.91 \pm 0.00^{\mathrm{a}}$ & $0.96 \pm 0.02^{\mathrm{b}}$ & $1.00 \pm 0.03^{\mathrm{b}}$ & $1.10 \pm 0.02^{\mathrm{c}}$ \\
\hline C14:0 & $37.71 \pm 0.01^{\mathrm{e}}$ & $122.30 \pm 0.11^{\mathrm{a}}$ & $69.89 \pm 0.08^{\mathrm{b}}$ & $50.15 \pm 0.02^{\mathrm{d}}$ & $58.73 \pm 0.03^{c}$ \\
\hline $\mathrm{C} 14: 1$ & $2.03 \pm 0.02^{\mathrm{d}}$ & $5.96 \pm 0.10^{\mathrm{a}}$ & $4.51 \pm 0.01^{\mathrm{b}}$ & $2.61 \pm 0.01^{\mathrm{c}}$ & $3.21 \pm 0.01^{\mathrm{c}}$ \\
\hline C15:0 & $12.22 \pm 0.02^{\mathrm{e}}$ & $35.31 \pm 0.03^{\mathrm{a}}$ & $21.45 \pm 0.01^{\mathrm{b}}$ & $17.18 \pm 0.02^{\mathrm{d}}$ & $17.92 \pm 0.04^{c}$ \\
\hline $\mathrm{C} 16: 0$ & $249.65 \pm 0.04^{\mathrm{d}}$ & $642.45 \pm 0.57^{\mathrm{a}}$ & $403.38 \pm 0.12^{\mathrm{b}}$ & $371.29 \pm 0.05^{\mathrm{c}}$ & $368.90 \pm 0.09^{c}$ \\
\hline $\mathrm{C} 16: 1$ & $204.13 \pm 0.02^{\mathrm{e}}$ & $593.66 \pm 0.33^{\mathrm{a}}$ & $354.40 \pm 0.08^{\mathrm{b}}$ & $272.41 \pm 0.30^{\mathrm{d}}$ & $298.22 \pm 0.11^{\mathrm{c}}$ \\
\hline C17:0 & $20.21 \pm 0.02^{\mathrm{e}}$ & $38.41 \pm 0.08^{\mathrm{a}}$ & $30.16 \pm 0.04^{\mathrm{b}}$ & $25.67 \pm 0.07^{\mathrm{d}}$ & $27.15 \pm 0.04^{\mathrm{c}}$ \\
\hline $\mathrm{C} 17: 1$ & $33.61 \pm 0.01^{\mathrm{e}}$ & $86.23 \pm 0.05^{\mathrm{a}}$ & $49.26 \pm 0.06^{\mathrm{b}}$ & $39.40 \pm 0.05^{\mathrm{d}}$ & $41.77 \pm 0.09^{c}$ \\
\hline C18:0 & $113.12 \pm 0.03^{\mathrm{e}}$ & $270.40 \pm 0.13^{\mathrm{a}}$ & $174.15 \pm 0.03^{\mathrm{c}}$ & $180.98 \pm 0.08^{\mathrm{b}}$ & $171.12 \pm 0.10^{\mathrm{d}}$ \\
\hline $\mathrm{C} 18: \ln 9 \mathrm{c}$ & $687.31 \pm 0.78^{\mathrm{d}}$ & $2112.33 \pm 1.70^{\mathrm{a}}$ & $1385.30 \pm 1.57^{\mathrm{b}}$ & $1304.53 \pm 0.94^{\mathrm{c}}$ & $1308.14 \pm 0.70^{\mathrm{c}}$ \\
\hline $\mathrm{C} 18: 2 \mathrm{n} 6 \mathrm{c}$ & $194.52 \pm 0.12^{\mathrm{e}}$ & $850.07 \pm 1.02^{\mathrm{a}}$ & $563.37 \pm 0.22^{\mathrm{b}}$ & $461.43 \pm 0.16^{\mathrm{c}}$ & $334.49 \pm 0.30^{\mathrm{d}}$ \\
\hline C20:0 & $2.20 \pm 0.00^{\mathrm{d}}$ & $14.13 \pm 0.03^{\mathrm{a}}$ & $9.60 \pm 0.07^{\mathrm{b}}$ & $5.62 \pm 0.04^{\mathrm{c}}$ & $4.55 \pm 0.02^{c}$ \\
\hline C18:3n6 & $3.23 \pm 0.02^{\mathrm{d}}$ & $7.39 \pm 0.10^{\mathrm{a}}$ & $5.31 \pm 0.05^{\mathrm{b}}$ & $3.48 \pm 0.02^{\mathrm{c}}$ & $3.12 \pm 0.03^{\mathrm{c}}$ \\
\hline $\mathrm{C} 18: 3 \mathrm{n} 3$ & $148.60 \pm 0.07^{\mathrm{e}}$ & $503.36 \pm 0.22^{\mathrm{a}}$ & $257.10 \pm 0.30^{\mathrm{b}}$ & $224.56 \pm 0.16^{\mathrm{d}}$ & $231.66 \pm 0.95^{c}$ \\
\hline C20:1 & $16.15 \pm 0.03^{\mathrm{d}}$ & $52.32 \pm 0.10^{\mathrm{a}}$ & $24.79 \pm 0.07^{\mathrm{b}}$ & $23.81 \pm 0.03^{c}$ & $24.02 \pm 0.06^{c}$ \\
\hline $\mathrm{C} 20: 2$ & $11.32 \pm 0.01^{\mathrm{e}}$ & $31.51 \pm 0.13^{\mathrm{a}}$ & $18.87 \pm 0.08^{\mathrm{b}}$ & $14.12 \pm 0.02^{\mathrm{d}}$ & $15.81 \pm 0.04^{\mathrm{c}}$ \\
\hline C20:3n 6 & $23.72 \pm 0.04^{\mathrm{d}}$ & $62.53 \pm 0.15^{\mathrm{a}}$ & $36.11 \pm 0.24^{\mathrm{b}}$ & $34.13 \pm 0.04^{\mathrm{c}}$ & $34.10 \pm 0.13^{c}$ \\
\hline $\mathrm{C} 20: 3 \mathrm{n} 3$ & $18.84 \pm 0.03^{\mathrm{e}}$ & $62.72 \pm 0.11^{\mathrm{a}}$ & $35.97 \pm 0.05^{\mathrm{b}}$ & $25.54 \pm 0.30^{\mathrm{d}}$ & $27.61 \pm 0.06^{\mathrm{c}}$ \\
\hline $\mathrm{C} 22: \ln 9$ & $201.32 \pm 0.10^{\mathrm{d}}$ & $366.21 \pm 0.81^{\mathrm{a}}$ & $298.85 \pm 0.42^{\mathrm{b}}$ & $270.15 \pm 0.63^{c}$ & $250.66 \pm 0.45^{c}$ \\
\hline $\mathrm{C} 20: 4 \mathrm{n} 6$ & $4.32 \pm 0.02^{\mathrm{d}}$ & $19.23 \pm 0.04^{\mathrm{a}}$ & $16.8 \pm 0.04^{\mathrm{b}}$ & $11.1 \pm 0.03^{\mathrm{c}}$ & $12.9 \pm 0.06^{\mathrm{c}}$ \\
\hline $\mathrm{C} 22: 2$ & $0.71 \pm 0.01^{\mathrm{d}}$ & $1.32 \pm 0.03^{\mathrm{a}}$ & $1.10 \pm 0.02^{\mathrm{b}}$ & $0.86 \pm 0.02^{\mathrm{c}}$ & $0.79 \pm 0.04^{\mathrm{c}}$ \\
\hline C20:5n3(EPA) & $229.65 \pm 0.47^{\mathrm{e}}$ & $543.21 \pm 1.28^{\mathrm{a}}$ & $360.78 \pm 1.55^{\mathrm{b}}$ & $307.72 \pm 0.13^{c}$ & $291.3 \pm 0.10^{\mathrm{d}}$ \\
\hline $\mathrm{C} 24: 0$ & $1.22 \pm 0.01^{\mathrm{c}}$ & $4.48 \pm 0.20^{\mathrm{a}}$ & $4.10 \pm 0.11^{\mathrm{a}}$ & $4.02 \pm 0.04^{\mathrm{a}}$ & $2.55 \pm 0.20^{\mathrm{b}}$ \\
\hline C24:1 & $11.91 \pm 0.04^{\mathrm{e}}$ & $17.72 \pm 0.13^{\mathrm{a}}$ & $16.25 \pm 0.07^{\mathrm{c}}$ & $17.02 \pm 0.09^{\mathrm{b}}$ & $15.30 \pm 0.03^{\mathrm{d}}$ \\
\hline C22:6n3(DHA) & $398.68 \pm 0.58^{\mathrm{e}}$ & $874.39 \pm 2.07^{\mathrm{a}}$ & $594.31 \pm 0.82^{\mathrm{b}}$ & $523.9 \pm 0.35^{\mathrm{c}}$ & $489.15 \pm 0.46^{\mathrm{d}}$ \\
\hline SFAs & $437.74 \pm 0.15^{\mathrm{d}}$ & $1131.26 \pm 3.32^{\mathrm{a}}$ & $712.12 \pm 4.15^{\mathrm{b}}$ & $660.89 \pm 1.67^{\mathrm{c}}$ & $665.08 \pm 1.09^{c}$ \\
\hline MUFAs & $1156.45 \pm 0.13^{\mathrm{e}}$ & $3233.31 \pm 5.40^{\mathrm{e}}$ & $2133.24 \pm 5.50^{\mathrm{e}}$ & $1929.87 \pm 2.72^{\mathrm{e}}$ & $1941.34 \pm 2.08^{\mathrm{e}}$ \\
\hline PUFAs & $1033.00 \pm 0.60^{\mathrm{e}}$ & $2954.59 \pm 5.45^{\mathrm{e}}$ & $1656.21 \pm 4.74^{\mathrm{e}}$ & $1714.19 \pm 2.14^{\mathrm{e}}$ & $1567.67 \pm 1.26^{\mathrm{e}}$ \\
\hline UFAs & $2189.45 \pm 0.41^{\mathrm{e}}$ & $6187.90 \pm 10.85^{\mathrm{a}}$ & $3789.45 \pm 10.24^{\mathrm{b}}$ & $3644.06 \pm 4.86^{\mathrm{c}}$ & $3509.01 \pm 3.34^{\mathrm{d}}$ \\
\hline
\end{tabular}

Data were expressed as mean \pm standard deviation of three determinations. Different lowercase letters in the same row indicate significant differences $(p<0.05)$. SFAs: total saturated fatty acids; UFAs: total unsaturated fatty acids; MUFAs: monounsaturated fatty acids; PUFAs: polyunsaturated fatty acids.

result of fish fillets grilled with various time intervals was also reported previously [19].

3.2. Sensory Scores, Fatty Acids, and Amino Acids of GSJs. Several researchers found that flavor and tenderness were the most important among various sensory attributes of jerky products. The hedonic test obtained scores, including appearance, flavor, texture, and whole acceptability of GSJs with different roasting times, are summarized in Table 1. It demonstrated that sensory outlook, flavor, and overall acceptability of GSJs increased from 20 to $60 \mathrm{~min}$, and then these quality profiles decreased to certain degree ( $80 \mathrm{~min}$ ).
The texture score increased when roasted up to $80 \mathrm{~min}$, because of moisture reduction, protein denaturation, and cross-linking, leading to the formation of crust rigidity of jerky products $[36,38]$. The present study recommended that 40-60 min of roasting was the optimum condition for production of GSJs, after weighing various sensory profiles.

Fatty acids can not only improve meat taste, but also offer calories and health-promoting polyunsaturated fatty acids for human body [40, 41]. Table 2 illustrates the overall fatty acid compositions of GSJs with different roasting times. As shown, both saturated fatty acids (SFAs) and unsaturated fatty acids (UFAs) of GSJs enhanced obviously after roasting $(p<0.05)$; especially, a jump was observed when GSJs 
TABLE 3: Amino acid profiles $\left(\mathrm{g} \cdot 100 \mathrm{~g}^{-1}\right)$ of giant salamander jerky as affected by roasting time.

\begin{tabular}{|c|c|c|c|c|c|}
\hline \multirow{2}{*}{ Amino acids } & \multicolumn{5}{|c|}{ Content $\left(\mathrm{g} \cdot 100 \mathrm{~g}^{-1}\right)$} \\
\hline & $0 \mathrm{~min}$ & $20 \mathrm{~min}$ & $40 \mathrm{~min}$ & $60 \mathrm{~min}$ & $80 \mathrm{~min}$ \\
\hline Aspartic acid (Asp) & $7.29 \pm 0.03^{\mathrm{a}}$ & $6.16 \pm 0.18^{\mathrm{b}}$ & $7.25 \pm 0.02^{\mathrm{a}}$ & $5.99 \pm 0.09^{\mathrm{b}}$ & $7.04 \pm 0.10^{\mathrm{a}}$ \\
\hline Threonine (Thr) & $3.45 \pm 0.05^{\mathrm{a}}$ & $2.92 \pm 0.02^{b}$ & $3.43 \pm 0.22^{\mathrm{a}}$ & $2.84 \pm 0.14^{\mathrm{b}}$ & $3.33 \pm 0.06^{\mathrm{a}}$ \\
\hline Serine (Ser) & $3.26 \pm 0.02^{\mathrm{a}}$ & $2.88 \pm 0.16^{\mathrm{b}}$ & $3.17 \pm 0.08^{\mathrm{a}}$ & $2.68 \pm 0.02^{\mathrm{b}}$ & $2.92 \pm 0.01^{b}$ \\
\hline Glutamic acid (Glu) & $11.3 \pm 0.15^{\mathrm{a}}$ & $9.65 \pm 0.09^{b}$ & $11.2 \pm 0.10^{\mathrm{a}}$ & $9.35 \pm 0.06^{\mathrm{b}}$ & $10.9 \pm 0.08^{\mathrm{a}}$ \\
\hline Glycine (Gly) & $4.08 \pm 0.01^{\mathrm{a}}$ & $4.24 \pm 0.03^{\mathrm{a}}$ & $3.68 \pm 0.03^{\mathrm{b}}$ & $3.38 \pm 0.07^{\mathrm{c}}$ & $3.70 \pm 0.04^{\mathrm{b}}$ \\
\hline Alanine (Ala) & $4.94 \pm 0.03^{\mathrm{a}}$ & $4.38 \pm 0.11^{\mathrm{b}}$ & $4.76 \pm 0.04^{\mathrm{a}}$ & $4.04 \pm 0.13^{c}$ & $4.82 \pm 0.07^{\mathrm{a}}$ \\
\hline Cysteine (Cys) & $0.70 \pm 0.10^{\mathrm{a}}$ & $0.69 \pm 0.04^{\mathrm{a}}$ & $0.65 \pm 0.02^{\mathrm{ab}}$ & $0.61 \pm 0.04^{\mathrm{b}}$ & $0.62 \pm 0.06^{\mathrm{b}}$ \\
\hline Valine (Val) & $3.40 \pm 0.05^{\mathrm{a}}$ & $2.87 \pm 0.02^{b}$ & $3.40 \pm 0.03^{\mathrm{a}}$ & $2.83 \pm 0.02^{\mathrm{b}}$ & $3.41 \pm 0.10^{\mathrm{a}}$ \\
\hline Methionine (Met) & $1.85 \pm 0.03^{\mathrm{b}}$ & $1.49 \pm 0.09^{c}$ & $1.96 \pm 0.22^{\mathrm{a}}$ & $1.54 \pm 0.10^{c}$ & $1.93 \pm 0.08^{\mathrm{a}}$ \\
\hline Isoleucine (Ile) & $3.44 \pm 0.04^{\mathrm{a}}$ & $2.87 \pm 0.17^{\mathrm{b}}$ & $3.46 \pm 0.05^{\mathrm{a}}$ & $2.84 \pm 0.06^{\mathrm{b}}$ & $3.42 \pm 0.09^{\mathrm{a}}$ \\
\hline Leucine (Leu) & $5.95 \pm 0.12^{\mathrm{a}}$ & $5.04 \pm 0.06^{\mathrm{b}}$ & $6.00 \pm 0.04^{\mathrm{a}}$ & $4.94 \pm 0.09^{\mathrm{b}}$ & $5.88 \pm 0.20^{\mathrm{a}}$ \\
\hline Tyrosine (Tyr) & $2.50 \pm 0.08^{\mathrm{a}}$ & $2.06 \pm 0.20^{\mathrm{b}}$ & $2.53 \pm 0.21^{\mathrm{a}}$ & $2.03 \pm 0.10^{\mathrm{b}}$ & $2.38 \pm 0.04^{c}$ \\
\hline Phenylalanine (Phe) & $3.08 \pm 0.02^{\mathrm{a}}$ & $2.59 \pm 0.04^{\mathrm{b}}$ & $3.08 \pm 0.08^{\mathrm{a}}$ & $2.58 \pm 0.12^{\mathrm{b}}$ & $2.98 \pm 0.19^{\mathrm{a}}$ \\
\hline Lysine (Lys) & $6.68 \pm 0.04^{\mathrm{a}}$ & $5.54 \pm 0.06^{\mathrm{c}}$ & $6.71 \pm 0.03^{\mathrm{a}}$ & $5.44 \pm 0.17^{c}$ & $6.31 \pm 0.05^{\mathrm{b}}$ \\
\hline Histidine (His) & $1.83 \pm 0.09^{\mathrm{a}}$ & $1.56 \pm 0.07^{\mathrm{b}}$ & $1.83 \pm 0.08^{\mathrm{a}}$ & $1.52 \pm 0.04^{\mathrm{b}}$ & $1.81 \pm 0.02^{\mathrm{a}}$ \\
\hline Arginine (Arg) & $4.72 \pm 0.07^{\mathrm{a}}$ & $4.35 \pm 0.20^{\mathrm{a}}$ & $4.63 \pm 0.16^{\mathrm{a}}$ & $3.84 \pm 0.04^{\mathrm{b}}$ & $4.54 \pm 0.06^{\mathrm{a}}$ \\
\hline Proline (Pro) & $3.07 \pm 0.13^{\mathrm{a}}$ & $3.03 \pm 0.08^{\mathrm{a}}$ & $2.86 \pm 0.04^{\mathrm{b}}$ & $2.67 \pm 0.09^{b}$ & $3.03 \pm 0.02^{\mathrm{a}}$ \\
\hline Tryptophan (Trp) & $0.23 \pm 0.02^{\mathrm{a}}$ & $0.20 \pm 0.01^{\mathrm{a}}$ & $0.19 \pm 0.05^{\mathrm{a}}$ & $0.21 \pm 0.02^{\mathrm{a}}$ & $0.20 \pm 0.03^{\mathrm{a}}$ \\
\hline TAA & $71.77 \pm 1.45^{\mathrm{a}}$ & $62.32 \pm 0.93^{\mathrm{b}}$ & $70.79 \pm 1.09^{\mathrm{a}}$ & $59.33 \pm 0.86^{\mathrm{b}}$ & $69.22 \pm 0.81^{\mathrm{a}}$ \\
\hline EAA & $31.40 \pm 0.00^{\mathrm{a}}$ & $26.54 \pm 0.00^{\mathrm{b}}$ & $31.39 \pm 0.00^{\mathrm{a}}$ & $25.94 \pm 0.00^{\mathrm{b}}$ & $30.76 \pm 0.00^{\mathrm{a}}$ \\
\hline
\end{tabular}

Data were expressed as mean \pm standard deviation of three determinations. Different lowercase letters in the same row indicate significant differences $(p<0.05)$. TAA: total amino acid; EAA (essential amino acids $)=\sum \mathrm{Lys}+\mathrm{Phe}+\mathrm{Val}+\mathrm{Ala}+\mathrm{Met}+\mathrm{Leu}+\mathrm{Ile}+\mathrm{Trp}+\mathrm{His}$.

roasted for $20 \mathrm{~min}$. Then, SFAs and UFAs were diminished significantly when roasting from 20 to $80 \mathrm{~min}$. Compared to raw (0 min), all GSJs after roasting showed higher content of SFAs and UFAs. SFAs in meat products would increase when subjected to a dry-heat cooking process, primarily because of oxidation of unsaturated bonds from fatty acids. C16:0 was found to be the relative more abundant saturated fatty acids (249.65-642.45 mg. $\left.100 \mathrm{~g}^{-1}\right)$ in GSJs, which was similar with previous reports about cooked orange-spotted grouper (Epinephelus coioides) [42], wild sea bream (Sparus aurata) [41], and roasted sardines (Sardinella brasiliensis) [43].

Table 2 also shows that GSJs contained more unsaturated fatty acids (2189.45-3509.01 mg. $100 \mathrm{~g}^{-1}$ ), including MUFAs (1156.45-1941.34 mg.100 g $\mathrm{g}^{-1}$ ) and PUFAs (1033.007-1567.67 mg.100 g $\mathrm{g}^{-1}$ ). Meanwhile, the contents of C20:5n3 (EPA) and C22:6n3 (DHA) in GSJs were relatively higher than other polyunsaturated fatty acids, which implied its potential health benefits correlated with many chronic cardiovascular symptoms [44]. The present study found that compared with raw (0 min), both MUFAs and PUFAs (including DHA and EPA) increased significantly $(p<0.05)$ when roasting for $20 \mathrm{~min}$ and then decreased significantly as roasting from 20 to $80 \mathrm{~min}$. Several literatures demonstrated that MUFAs and PUFAs of meat and aquatic products would be promptly destroyed after thermal processing [42, 44, 45]. Although the content of MUFAs and PUFAs in GSJs decreased significantly with extension of roasting time, GSJs after roasting still showed higher unsaturated fatty acids (including DHA and EPA) than raw control (0 min), perhaps owing to giant salamander's high unsaturated fatty acids content. The present fatty acid profile results were in agreement with previous reports, such as pork, lamb, sardines, and sea bream after dry-heat cooking process [41, 43-46].
Therefore, GSJs might be considered as a novel jerky product with great amount of healthy unsaturated fatty acids.

Table 3 shows the amino acid compositions of GSJs with different roasting times, as well as total essential amino acids (TEAAs) and total amino acids (TAAs). As shown, roasting process could fluctuate the amino acid profile of GSJs to certain extent, chiefly owing to protein and lipid oxidation during roasting $[17,19,20]$. The most abundant amino acids in GSJs were glutamic acid $\left(9.35-11.3 \mathrm{~g} \cdot 100 \mathrm{~g}^{-1}\right)$, aspartic acid $\left(5.99-7.29 \mathrm{~g} \cdot 100 \mathrm{~g}^{-1}\right)$, lysine $\left(5.44-6.71 \mathrm{~g} \cdot 100 \mathrm{~g}^{-1}\right)$, leucine (4.94-6.00 g.100 g $\mathrm{g}^{-1}$ ), and alanine (4.04-4.94 g.100 g $\left.{ }^{-1}\right)$. The total amino acids and essential amino acids were among $59.33-71.77 \mathrm{~g} \cdot 100 \mathrm{~g}^{-1}$ and $25.94-31.40 \mathrm{~g} \cdot 100 \mathrm{~g}^{-1}$, respectively, which was higher than claw meat (Eriocheir sinensis) [40]. Therefore, GSJs could also be considered as a nutritive jerky product with balanced amino acid compositions.

\subsection{SEM Microstructure of GSJs during Roasting Process.} To further understand the muscular tissue during roasting, the SEM photo of GSJs with different roasting times was captured and is shown in Figure 2. As can be seen, the muscle fibers of GSJs experience certain aggregation when roasted from 0 to $20 \mathrm{~min}$, and then suffered to some extent depolymerization and fracture when roasted from 40 to $80 \mathrm{~min}$, which could cause corresponding texture and sensory quality changes of jerky during roasting. This phenomenon was mainly owing to initial protein denaturation, shrinkage, and water evaporation caused by heat transfer during roasting [36-39].

3.4. LF-NMR and MRI Signals as Affected by Roasting Time. Figure 3 exhibits the profile of $T_{2}$ relaxation spectrum acquired by polyexponential simulating of constant allotted 


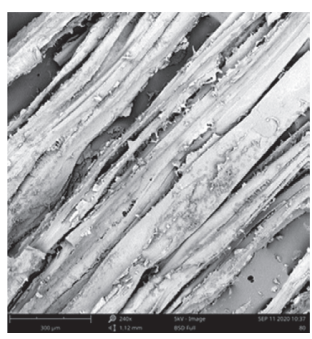

(a)

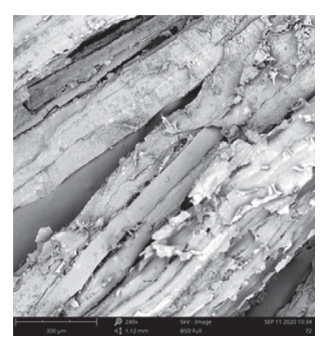

(b)

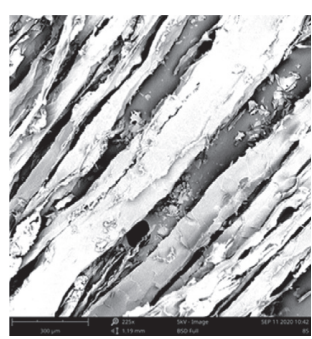

(c)

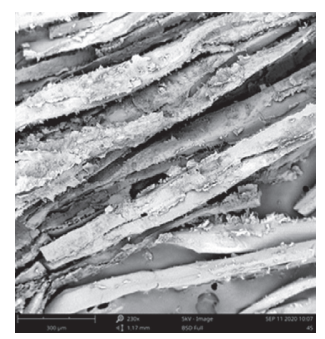

(d)

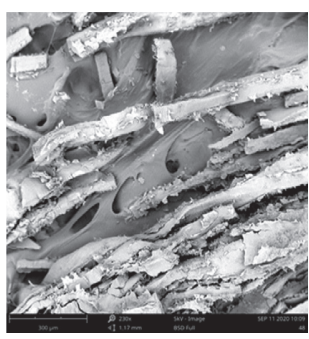

(e)

FIgURE 2: Microstructure observation of GSJs during roasting through scanning electron microscopy (SEM) at a magnification of 230x. (a) 0 min. (b) $20 \mathrm{~min}$. (c) $40 \mathrm{~min}$. (d) $60 \mathrm{~min}$. (e) $80 \mathrm{~min}$.

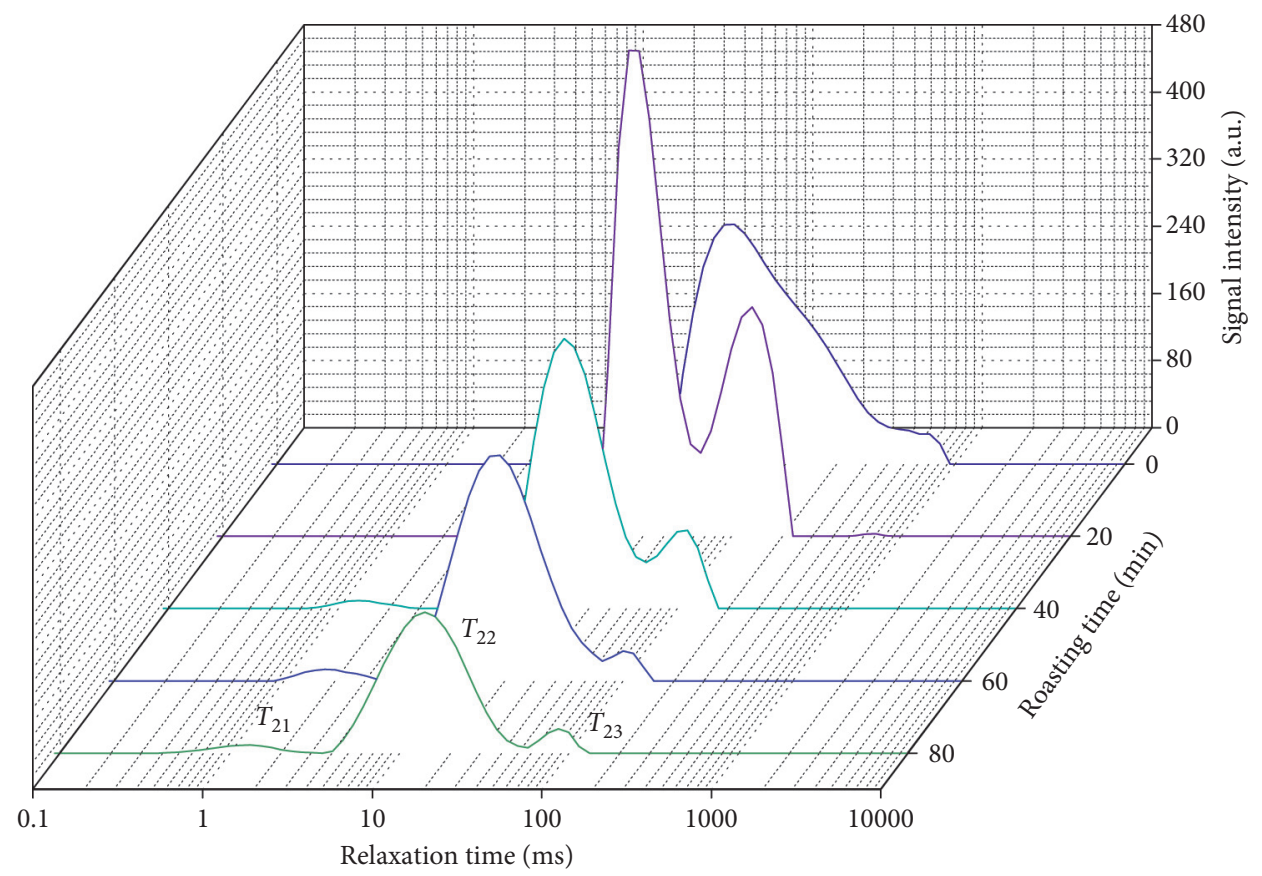

FIGURE 3: Characteristic LF-NMR $T_{2}$ relaxation spectra of giant salamander jerky as affected by roasting times.

CPMG spectra for GSJs with different roasting time. Before roasting $(0 \mathrm{~min})$, the curve signal showed two major groups with the $T_{2}$ time around 65.06 and $613.59 \mathrm{~ms}$. But after roasting for $20 \mathrm{~min}$, the third group with the $T_{2}$ time below $10 \mathrm{~ms}$ appeared in grilled jerky products. The first peak $T_{21}$ with the minimum time below $10 \mathrm{~ms}$, generally, is considered as bound moisture, which is closely combined with nearby substances. The second peak $T_{22}$ around $10-100 \mathrm{~ms}$ is thought to be immobilized moisture captured in meat lattice. The third peak $T_{23}$ with the maximum time around $100-1000 \mathrm{~ms}$ is deemed to bulk moisture [24, 25].

Figure 3 shows that the $T_{2}$ of bound, immobilized, and bulk moisture of GSJs all diminished after roasting from 20 to $80 \mathrm{~min}$, which implied the reduction of water mobility. The quantitative data of $T_{21}, T_{22}$, and $T_{23}$ and corresponding peak areas $A_{21}, A_{22}$, and $A_{23}$ of GSJs among various roasting intervals are shown in Table 4 . The $T_{21}$ diminished from $4.08 \mathrm{~ms}$ for GSJs roasted $20 \mathrm{~min}$, to $2.87 \mathrm{~ms}$ for $40 \mathrm{~min}$, $1.73 \mathrm{~ms}$ for $60 \mathrm{~min}$, and $1.04 \mathrm{~ms}$ for $80 \mathrm{~min}$. With the roasting time extending $(20-80 \mathrm{~min}), T_{22}$ decreased from
$65.06 \mathrm{~ms}$ to $14.17 \mathrm{~ms}(p<0.05)$, and $T_{23}$ decreased from $613.59 \mathrm{~ms}$ to $86.97 \mathrm{~ms}(p<0.05)$, respectively.

As the peak proportion of $T_{2}$ components generally is directly correlated with moisture level in food products, the present data demonstrated that the proportion of bound moisture $A_{21}$ enhanced from 11.95 to $72.06(p<0.05)$, the proportion of immobilized moisture $A_{22}$ decreased from 3933.56 to 1622.75 , and the proportion of bulk moisture $A_{23}$ enhanced from 109.31 to $598.19(p<0.05)$ and then decreased from 598.19 to 100.80 . It can be seen that these NMR signals illustrated that the movabilities of bound, immobilized, and bulk moisture for GSJs were shrunk to some extent, mainly owing to the moisture evaporation loss during roasting process $[25,27]$. A similar result of Pacific oyster after hot-air dehydrating at various time intervals was also reported previously [24].

Figure 4(a) shows the moisture allotment of GSJs by MRI through the proton density weighted photos, obtained during the roasting process for each sample. The red region denotes high proton accumulation, and the blue region 
TABLE 4: $T_{2}$ relaxation time of Chinese giant salamander jerky as affected by roasting time.

\begin{tabular}{|c|c|c|c|c|c|c|}
\hline Roasting time (min) & $T_{21}(\mathrm{~ms})$ & $T_{22}(\mathrm{~ms})$ & $T_{23}(\mathrm{~ms})$ & $A_{21}$ & $A_{22}$ & $A_{23}$ \\
\hline 0 & - & $65.06 \pm 1.52^{\mathrm{a}}$ & $613.59 \pm 8.45^{\mathrm{a}}$ & - & $3933.56 \pm 44.85^{\mathrm{a}}$ & $109.31 \pm 4.62^{\mathrm{d}}$ \\
\hline 20 & $4.08 \pm 0.16^{\mathrm{a}}$ & $50.11 \pm 2.35^{\mathrm{b}}$ & $194.20 \pm 10.04^{\mathrm{b}}$ & $11.95 \pm 1.06^{\mathrm{d}}$ & $3110.32 \pm 36.07^{b}$ & $598.19 \pm 11.07^{\mathrm{a}}$ \\
\hline 40 & $2.87 \pm 0.10^{\mathrm{b}}$ & $41.24 \pm 1.88^{\mathrm{c}}$ & $137.90 \pm 5.77^{\mathrm{c}}$ & $31.56 \pm 2.85^{\mathrm{c}}$ & $2559.40 \pm 24.30^{\mathrm{c}}$ & $430.24 \pm 3.25^{\mathrm{b}}$ \\
\hline 60 & $1.73 \pm 0.22^{\mathrm{C}}$ & $32.10 \pm 0.70^{\mathrm{d}}$ & $95.21 \pm 2.72^{\mathrm{d}}$ & $55.04 \pm 1.47^{\mathrm{b}}$ & $2678.68 \pm 10.22^{\mathrm{d}}$ & $113.53 \pm 1.04^{\mathrm{c}}$ \\
\hline 80 & $1.04 \pm 0.14^{\mathrm{d}}$ & $14.17 \pm 1.05^{\mathrm{e}}$ & $86.97 \pm 1.03^{\mathrm{e}}$ & $72.06 \pm 2.07^{\mathrm{a}}$ & $1622.75 \pm 16.47^{\mathrm{e}}$ & $100.80 \pm 2.47^{\mathrm{d}}$ \\
\hline
\end{tabular}

Data were expressed as mean \pm standard deviation of three determinations. Different lowercase superscripts in the same column denote significant differences $(p<0.05)$.

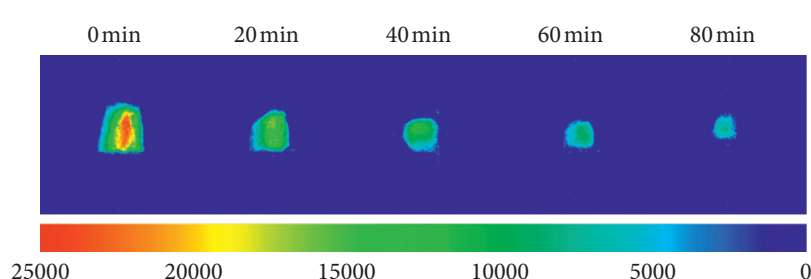

(a)

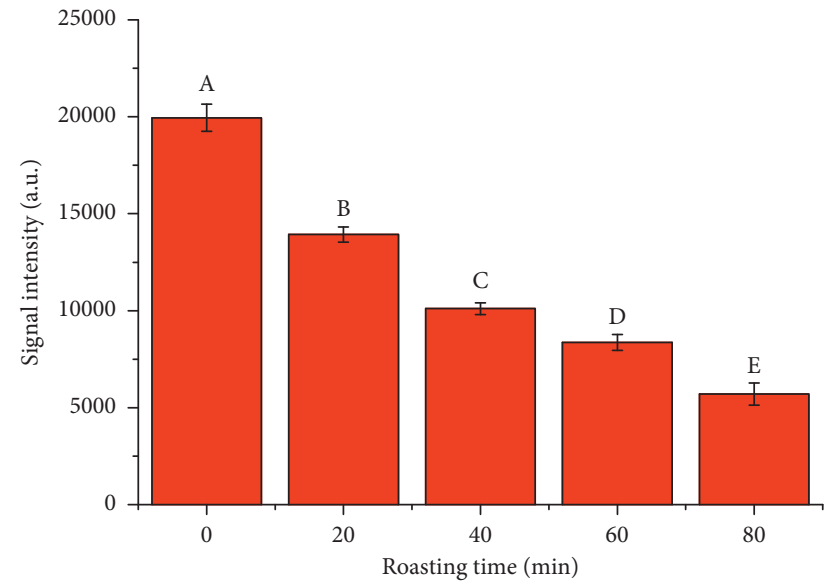

(b)

FIgURe 4: Proton density weighted magnetic resonance images of giant salamander jerky with different roasting times (a) and the corresponding histogram of quantitative signal intensity (b).

TABLE 5: Correlation coefficient among LM-NMR signal, moisture content, water activity, yield, shear force, and overall acceptability.

\begin{tabular}{lccccccc}
\hline & $T_{22}$ & $T_{23}$ & MRI signal & Moisture content & Yield & Shear force & Overall acceptability \\
\hline$T_{22}$ & 1 & $0.974^{* *}$ & $0.982^{* *}$ & $0.982^{* *}$ & $0.988^{* *}$ & $-0.913^{* *}$ & -0.503 \\
$T_{23}$ & & 1 & $0.966^{* *}$ & $0.967^{* *}$ & $0.964^{* *}$ & $-0.887^{* *}$ & $-0.671^{*}$ \\
MRI signal & & & 1 & $0.980^{* *}$ & $0.996^{* *}$ & $-0.965^{* *}$ & $-0.672^{*}$ \\
Moisture content & & & & 1 & $0.983^{* *}$ & $-0.916^{* *}$ & $-0.588^{*}$ \\
Yield & & & & & $-0.961^{* *}$ & -0.571 \\
Shear force & & & & & & 1 & $0.635^{*}$ \\
Overall acceptability & & & & & & 1 \\
\hline
\end{tabular}

** Significant correlation at 0.01 (two-tailed). ${ }^{*}$ Significant correlation at 0.05 (two-tailed).

denotes low proton accumulation. With the prolongation of roasting time, a steady diminishment was noticed by the proton signals of brighter proportion, implying the drop of longer $T_{2}$ signal of moisture after roasting $[24,27]$. In addition, the shrinkage of proton signal from outer surface to inward district can be found clearly. Meantime, the red district almost disappeared for GSJs after $80 \mathrm{~min}$ roasting with $26.44 \%$ water proportion, implying low proton accumulation. This phenomenon was because of the water evaporation caused by heat transfer during roasting [16, 19]. Figure 4(b) depicts the quantitative proton accumulation of Figure 4(a), which shrunk obviously during roasting. These results were in agreement with previous reports about dried abalone at different drying times [25].
3.5. Correlation Analysis of Various Parameters. Correlation analysis between the quality characteristics and NMR relaxation parameters was performed by bivariate analysis Pearson's correlation coefficient, and the results showed that the relaxation time $T_{22}, T_{23}$ and quality attributes displayed excellent correlations as shown in Table 5.

The corresponding correlation coefficients of $T_{22}$ and $T_{23}$ were positively correlated with MRI signal, moisture content, and yield of GSJs, but negatively correlated with shear force and overall acceptability, respectively (Table 5). This result demonstrates that the immobilized water and free water are the main form of the moisture and determines the changes of quality characteristic of GSJs during the roasting process $[19,24]$. Thus, the LF-NMR data could be applied to 
predict the moisture content and quality characteristics of GSJs during the roasting process as a fast and nondestructive method [32, 47].

\section{Conclusion}

In summary, the $a^{*}, b^{*}$ values and shear force of giant salamander jerkies (GSJs) increased when roasting from 20 to $80 \mathrm{~min}$, while $L^{*}$, yield, and moisture content decreased significantly $(p<0.05)$. Sensory assessment showed the whole acceptability of GSJs at a roasting time of 40-60 min had higher scores. GSJs contained great amount of healthy unsaturated fatty acids (including DHA and EPA), and the total amino acids and essential amino acids were among 59.33-71.77 g. $100 \mathrm{~g}^{-1}$ and $25.94-31.40 \mathrm{~g} \cdot 100 \mathrm{~g}^{-1}$, respectively. The mobility of the immobilized and bulk moisture was shrunk dramatically after roasting. The proton density weighted photos also depicted the moisture diminishment during roasting. In addition, $T_{22}$ and $T_{23}$ were positively correlated with MRI signal, moisture content, and yield of GSJs, but negatively correlated with shear force and overall acceptability, respectively. Thus, in view of various quality attributes and sensory evaluation, a roasting time of 40-60 min was favored for nutritive GSJs production, and LF-NMR and MRI might be employed to profile the quality characteristics during roasting as a rapid and nondestructive analytical tool.

\section{Data Availability}

All data generated or analysed during this study are included in this article.

\section{Conflicts of Interest}

The authors declare that there are no conflicts of interest regarding the publication of this study.

Wengang Jin and Xiaohua Chen contributed equally to this work.

\section{Authors' Contributions}

Wengang Jin and Xiaohua Chen conducted investigation, wrote the original draft, and performed plot analysis. Jingzhang Geng performed partial analysis and visualization. Jing Jin, Jinjin Pei, and Dejing Chen collected resources and tested amino acid and fatty acid content. Ruichang Gao reviewed and edited the manuscript, supervised the work, and acquired funding.

\section{Acknowledgments}

This study was mainly funded by Collaborative Innovation Center Project for Qin-Ba Mountain Bio-Resources Utilization and Development (no. QBXT-18-04) and China Scholarship Council (no. 202008610071), and partial fund from Shaanxi "Sanqin Scholar" Innovation Team was greatly appreciated.

\section{References}

[1] X. Geng, J. Guo, X. Zang et al., "Proteomic analysis of eleven tissues in the Chinese giant salamander (Andrias davidianus)," Scientific Reports, vol. 9, no. 1, pp. 1-10, 2019.

[2] C. Lu, J. Chai, R. W. Murphy, and J. Che, "Giant salamanders: farmed yet endangered," Science, vol. 367, no. 6481, p. 989, 2020.

[3] D. He, W. Zhu, W. Zeng et al., "Nutritional and medicinal characteristics of Chinese giant salamander (Andrias davidianus) for applications in healthcare industry by artificial cultivation: a review," Food Science and Human Wellness, vol. 7, no. 1, pp. 1-10, 2018.

[4] W.-G. Jin, J. Pei, Y.-N. Du et al., "Characterization and functional properties of gelatin extracted from Chinese giant salamander (Andrias davidianus) skin," Journal of Aquatic Food Product Technology, vol. 28, no. 8, pp. 861-876, 2019.

[5] A. H. Ramadhan, T. Nawas, X. W. Zhang, W. M. Pembe, W. S. Xia, and Y. S. Xu, "Purification and identification of a novel antidiabetic peptide from Chinese giant salamander (Andrias davidianus) protein hydrolysate against $\alpha$-amylase and $\alpha$-glucosidase," International Journal of Food Properties, vol. 20, pp. s3360-s3372, 2018.

[6] J. Pei and L. Jiang, "Antimicrobial peptide from mucus of Andrias davidianus: screening and purification by magnetic cell membrane separation technique," International Journal of Antimicrobial Agents, vol. 50, no. 1, pp. 41-46, 2017.

[7] J. Pei, D. Chen, W. Jin et al., "Structure and mode of action of a novel antibacterial peptide from the blood of Andrias davidianus," Letters in Applied Microbiology, vol. 69, no. 5, pp. 312-317, 2019.

[8] Y. F. Hu, N. N. Li, J. R. Chen, Y. S. Jia, J. Liang, and Y. F. Li, "Effect of chlorine dioxide on quality of giant salamander cutting meats in small modified atmosphere packaging," Advance Journal of Food Science and Technology, vol. 10, no. 4, pp. 302-308, 2016.

[9] W. Jin, P. Zhao, J. Jin et al., "Volatile component fingerprint analysis of giant salamander (Andrias davidiauns) from different edible parts based on gas chromatography-ion mobility spectroscopy," Food Science, 2021.

[10] J. Luo, X. Yang, Y. Cao, G. Li, Y. Meng, and C. Li, "Structural characterization and in vitro immunogenicity evaluation of amphibian-derived collagen type II from the cartilage of Chinese Giant Salamander (Andrias davidianus)," Journal of Biomaterials Science, Polymer Edition, vol. 31, no. 15, pp. 1941-1960, 2020.

[11] J. Ren, Y. Song, H. Kang et al., "Optimization of processing parameters for collagen extraction from Chinese giant salamander (Andrias davidianus) skin after dense phase carbon dioxide pretreatment," Food Science, vol. 38, no. 16, pp. 198-204, 2017.

[12] J. Pan, H. Lian, M. Shang et al., "Physicochemical properties of Chinese giant salamander (Andrias davidianus) skin gelatin as affected by extraction temperature and in comparison with fish and bovine gelatin," Journal of Food Measurement and Characterization, vol. 14, no. 5, pp. 2656-2666, 2020.

[13] W. Zhu, Y. Ji, Y. Wang et al., "Structural characterization and in vitro antioxidant activities of chondroitin sulfate purified from Andrias davidianus cartilage," Carbohydrate Polymers, vol. 196, pp. 398-404, 2018.

[14] L. Li, L. Zan, and Z. Ren, "Fatty acid composition, and antioxidant and rheological behavior of Chinese giant 
salamander (Andrias davidianus) oil," Journal of Chinese Cereal Oil Association, vol. 9, pp. 82-86, 2009.

[15] D. Ni, Z. He, D. Zhang, and D. Deng, "Changes in nutritional quality during processing of giant salamander soup," Food Science, vol. 38, no. 20, pp. 119-224, 2017.

[16] T.-K. Kim, H.-W. Kim, Y.-Y. Lee, H. W. Jang, Y.-B. Kim, and Y.-S. Choi, "Quality characteristics of duck jerky: combined effects of collagen and konjac," Poultry Science, vol. 99, no. 1, pp. 629-636, 2020.

[17] F. A. P. Silva, M. Estévez, V. C. S. Ferreira et al., "Protein and lipid oxidations in jerky chicken and consequences on sensory quality," LWT, vol. 97, pp. 341-348, 2018.

[18] D.-J. Han, J.-Y. Jeong, J.-H. Choi et al., "Effects of curing methods on the quality characteristics of pork jerky," Food Science and Technology Research, vol. 17, no. 3, pp. 179-186, 2011.

[19] L. Cai, S. Ma, X. Cheng, A. Cao, J. Feng, and J. Li, "Effects of different roasting time periods on character and flavor in sardine slices," Journal of Fisheries of China, vol. 40, no. 5, pp. 785-798, 2016.

[20] F. A. P. Silva, V. C. S. Ferreira, M. S. Madruga, and M. Estévez, "Effect of the cooking method (grilling, roasting, frying and sous-vide) on the oxidation of thiols, tryptophan, alkaline amino acids and protein cross-linking in jerky chicken," Journal of Food Science and Technology, vol. 53, no. 8, pp. 3137-3146, 2016.

[21] S. M. Goi and V. O. Salvador, "'Prediction of cooking times and weight losses during meat roasting," Journal of Food Engineering, vol. 100, no. 1, pp. 1-11, 2010.

[22] W. S. Chen, D. C. Liu, and M. T. Chen, "The effect of roasting temperature on the formation of volatile compounds in Chinese-style pork jerky," Asian-Australasian Journal of Animal Sciences, vol. 15, no. 3, pp. 427-431, 2002.

[23] L. Liu, Q. Liu, B. Kong, X. Wang, L. Liu, and J. Han, "Effect of deep-frying temperature on moisture distribution and quality of beef jerky based on LF-NMR," Journal of Chinese Institute of Food Science and Technology, vol. 16, no. 5, pp. 238-244, 2016.

[24] S. Cheng, T. Zhang, L. Yao et al., "Use of low-field-NMR and MRI to characterize water mobility and distribution in pacific oyster (Crassostrea gigas) during drying process," Drying Technology, vol. 36, no. 5, pp. 630-636, 2018.

[25] Y. Song, X. Zang, T. Kamal et al., "Real-time detection of water dynamics in abalone (Haliotis discus hannai Ino) during drying and rehydration processes assessed by LF-NMR and MRI," Drying Technology, vol. 36, no. 1, pp. 72-83, 2018.

[26] E. Kirtil and M. H. Oztop, " $1 \mathrm{H}$ nuclear magnetic resonance relaxometry and magnetic resonance imaging and applications in food science and processing," Food Engineering Reviews, vol. 8, no. 1, pp. 1-22, 2016.

[27] T. Kamal, Y. Song, T. Zhang, B. W. Zhu, and M. Tan, "Potential uses of LF-NMR and MRI in the study of water dynamics and quality measurement of fruits and vegetables," Journal of Food Processing and Preservation, vol. 43, no. 11, pp. 1974-1987, 2019.

[28] R. Lin, S. Cheng, S. Wang, M. Tan, and B. Zhu, "Influence of refrigerated storage on water status, protein oxidation, microstructure, and physicochemical qualities of atlantic mackerel (Scomber scombrus)," Foods, vol. 10, no. 2, p. 214, 2021.

[29] M. Li, H. Wang, G. Zhao et al., "Determining the drying degree and quality of chicken jerky by LF-NMR," Journal of Food Engineering, vol. 139, pp. 43-49, 2014.
[30] T.-H. Kim, Y.-S. Choi, J.-H. Choi et al., "Physicochemical properties and sensory characteristics of semi-dried pork jerky with rice bran fiber," Korean Journal for Food Science of Animal Resources, vol. 30, no. 6, pp. 966-974, 2010.

[31] H.-S. Yang, Y.-H. Hwang, S.-T. Joo, and G.-B. Park, "The physicochemical and microbiological characteristics of pork jerky in comparison to beef jerky," Meat Science, vol. 82, no. 3, pp. 289-294, 2009.

[32] W. Jin, J. Pei, S. Wang, X. Chen, R. Gao, and M. Tan, "Effect of continuous and intermittent drying on water mobility of fresh walnuts (Juglans regia L.): a LF-NMR study," Drying Technology, pp. 1-11, 2020.

[33] V. Gök, E. Obuz, and L. Akkaya, "Effects of packaging method and storage time on the chemical, microbiological, and sensory properties of Turkish pastirma-a dry cured beef product," Meat Science, vol. 80, no. 2, pp. 335-344, 2008.

[34] D. Pu, Y. Shan, W. Duan et al., "Characterization of the key aroma compounds in the fruit of Litsea pungens Hemsl. (LPH) by GC-MS/O, OAV, and Sensory Techniques," Journal of Food Quality, vol. 2021, Article ID 6668606, 9 pages, 2021.

[35] A. M. Elansari and A. I. Hobani, "Effect of temperature and moisture content on thermal conductivity of four types of meat," International Journal of Food Properties, vol. 12, no. 2, pp. 308-315, 2009.

[36] S. Barbut, "Frying - effect of coating on crust microstructure, color, and texture of lean meat portions," Meat Science, vol. 93, no. 2, pp. 269-274, 2013.

[37] S. Barbut, "Microstructure, texture and colour development during crust formation on whole muscle chicken fillets," British Poultry Science, vol. 54, no. 2, pp. 176-182, 2013.

[38] P. Konieczny, J. Stangierski, and J. Kijowski, "Physical and chemical characteristics and acceptability of home style beef jerky," Meat Science, vol. 76, no. 2, pp. 253-257, 2007.

[39] M. Nakamura, W. Mao, M. Fukuoka, and N. Sakai, "Analysis of the color change in fish during the grilling process," Food Science and Technology Research, vol. 17, no. 6, pp. 471-478, 2011.

[40] Y.-R. Guo, S.-Q. Gu, X.-C. Wang, L.-M. Zhao, and J.-Y. Zheng, "Comparison of fatty acid and amino acid profiles of steamed Chinese mitten crab," Fisheries Science, vol. 80, no. 3, pp. 621-633, 2014.

[41] A. Mnari Bhouri, H. Jrah Harzallah, M. Dhibi, I. Bouhlel, M. Hammami, and A. Chaouch, "Nutritional fatty acid quality of raw and cooked farmed and wild sea bream (Sparus aurata)," Journal of Agricultural and Food Chemistry, vol. 58, no. 1, pp. 507-512, 2010.

[42] Z. Momenzadeh, A. Khodanazary, and K. Ghanemi, "Effect of different cooking methods on vitamins, minerals and nutritional quality indices of orange-spotted grouper (Epinephelus coioides)," Journal of Food Measurement and Characterization, vol. 11, no. 2, pp. 434-441, 2017.

[43] M. K. Bulla, J. I. Simionato, M. Matsushita et al., "Proximate composition and fatty acid profile of raw and roasted saltdried sardines (Sardinella Brasiliensis)," Food and Nutrition Sciences, vol. 2, no. 5, pp. 440-443, 2011.

[44] M. R. Neff, S. P. Bhavsar, E. Braekevelt, and M. T. Arts, "Effects of different cooking methods on fatty acid profiles in four freshwater fishes from the Laurentian Great Lakes region," Food Chemistry, vol. 164, no. 4, pp. 544-550, 2014.

[45] M. M. Campo, E. Muela, J. L. Olleta et al., "Influence of cooking method on the nutrient composition of Spanish light lamb," Journal of Food Composition and Analysis, vol. 31, no. 2, pp. 185-190, 2013. 
[46] R. Wang, Q. Wang, W. Jiang, J. Chen, X. Ye, and D. Liu, "Effects of cooking methods on lipid oxidation and fatty acid profiles in intramuscular tissues of pork," Journal of Chinese Institute of Food Science and Technology, vol. 17, no. 7, pp. 61-68, 2017.

[47] T. Kamal, Y. Song, Z. Tan, B.-W. Zhu, and M. Tan, "Effect of hot-air oven dehydration process on water dynamics and microstructure of apple (Fuji) cultivar slices assessed by LFNMR and MRI," Drying Technology, vol. 37, no. 15, pp. 1974-1987, 2019. 